\title{
On the Direct Evaluation of Surface Integral Equation Impedance Matrix Elements Involving Point Singularities
}

\author{
Athanasios G. Polimeridis and Juan R. Mosig, Fellow, IEEE
}

\begin{abstract}
The direct evaluation method tailored to the 4-D singular integrals over vertex adjacent triangles, arising in the first-kind and second-kind Fredholm surface integral equation formulations, is presented. A combination of singularity cancellation, reordering of the integrations, and one analytical integration results in 3-D integrals of sufficiently smooth functions, allowing a straightforward computation by standard Gaussian rules. Numerical results demonstrate that the uncertainty about the accuracy of the impedance matrix elements associated to the interaction of vertex adjacent triangles is safely removed.
\end{abstract}

Index Terms-Computational electromagnetics, method of moments (MoM), singular integrals, singularity cancellation, surface integral equations (SIEs).

\section{INTRODUCTION}

$\mathbf{S}$ TATE-OF-THE-ART surface integral equation (SIE) formulations, numerically solved by means of Galerkin method of moments (MoM), necessitate an accurate and efficient computation of the associated impedance matrix elements. In the case of disjoint supports of basis and testing functions, the arising multidimensional integrals are regular, allowing a straightforward numerical integration. Hence, special emphasis is naturally laid upon the most challenging cases that appear when those supports are overlapping or share some common points, thus giving rise to singular integrals. More specifically, the focal point of this work is the class of multidimensional integrals involving point singularities. Literally, the singular integrals that stem from MoM SIE formulations can be categorized into $1 / R$ weakly singular (improper Riemann integrable) and $1 / R^{2}$ strongly singular (Cauchy), provided certain restrictions to both basis and testing functions.

It is also true that those 4-D singular integrals have been under scrutiny by numerous researchers over the last decades. Among the various contributions presented so far, the singularity cancellation method is deemed to hold a workhorse status (interested readers could consult the seminal publications [1] and [2] for a more detailed overview of previous work relevant to this method). In fact, although recent advances have clearly demonstrated that the inner (potential) 2-D integrals can be computed to any prescribed precision [3], there are still some shortcomings that need to be addressed. For instance, it is not clear if this accuracy can be preserved for the actual impedance matrix ele-

Manuscript received April 05, 2011; revised May 24, 2011; accepted June 07, 2011. Date of publication June 13, 2011; date of current version July 05, 2011. This work was supported by the COST Actions IC0803-RFCSET and IC0603-ASSIST.

The authors are with the Laboratory of Electromagnetics and Acoustics (LEMA), Ecole Polytechnique Fédérale de Lausanne (EPFL), 1015 Lausanne, Switzerland (e-mail: athanasios.polymeridis@epfl.ch).

Digital Object Identifier 10.1109/LAWP.2011.2159469 ments, i.e., the final 4-D singular integrals. Moreover, the vast majority of the singularity cancellation techniques can solely handle the weakly singular kernels. Therefore, in cases where both first-kind and second-kind Fredholm SIE formulations are present, the accuracy of the associated impedance matrix elements is critically contaminated.

In an attempt to overcome the reported drawbacks and meet the requirements for an accurate and efficient computation of the SIE impedance matrix elements, the authors have recently extended the pioneering work of Gray et al. [4], [5]. The resulting direct evaluation method tailored to the most demanding weakly singular and strongly singular integrals (e.g., coincident and edge adjacent triangles in the case of typical triangular discretization schemes) was presented in [6]-[8], respectively. Based on the preliminary results presented in [7], it is clear that the case of 4-D integrals involving point singularities (e.g., vertex adjacent triangles) is not to be overlooked, as a careless straightforward treatment may introduce significant numerical error.

This letter presents the direct evaluation method tailored to the SIE impedance matrix elements involving point singularities and linear basis/testing functions. Both weakly singular and strongly singular kernels are treated in a systematic way. The final algorithms clearly outperform direct integration approaches, as shown by relevant numerical experiments, and can guarantee numerically exact results (to be defined) in very reasonable run times. This letter puts a closure on the direct evaluation of singular integrals arising in traditional SIE formulations. Further generalizations for high-order and/or singular basis functions defined on arbitrary curvilinear elements is a matter of future investigations.

\section{Problem Statement}

The evaluation of the MoM impedance matrix elements in well-tested SIE, using RWG basis functions $\mathbf{f}$ and either RWG or $\hat{\mathbf{n}} \times$ RWG testing functions $\mathbf{g}$ [9], calls for the computation of the following 4-D singular integrals:

$$
\begin{aligned}
I_{1} & :=\int_{E_{P}} \nabla \cdot \mathbf{f}(\mathbf{r}) \int_{E_{Q}} G\left(\mathbf{r}, \mathbf{r}^{\prime}\right) \nabla^{\prime} \cdot \mathbf{f}\left(\mathbf{r}^{\prime}\right) d S^{\prime} d S \\
I_{2} & :=\int_{E_{P}} \mathbf{f}(\mathbf{r}) \cdot \int_{E_{Q}} G\left(\mathbf{r}, \mathbf{r}^{\prime}\right) \mathbf{f}\left(\mathbf{r}^{\prime}\right) d S^{\prime} d S \\
I_{3} & :=\int_{E_{P}} \mathbf{g}(\mathbf{r}) \cdot \int_{E_{Q}} \nabla G\left(\mathbf{r}, \mathbf{r}^{\prime}\right) \times \mathbf{f}\left(\mathbf{r}^{\prime}\right) d S^{\prime} d S
\end{aligned}
$$

which incorporate the free-space Green's function

$$
G\left(\mathbf{r}, \mathbf{r}^{\prime}\right)=G(R)=\frac{e^{-j k R}}{R}
$$




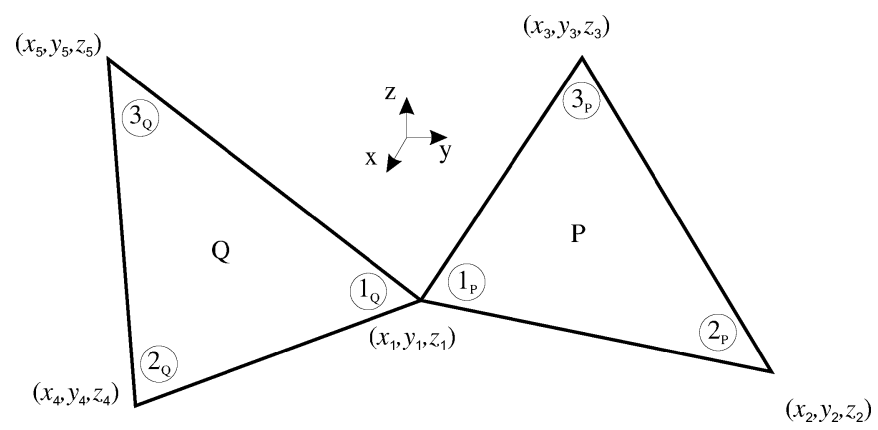

Fig. 1. Orientation of the triangular elements in the vertex adjacent case.

with $R=\left|\mathbf{r}-\mathbf{r}^{\prime}\right|$ being the distance function and $k=\omega \sqrt{\varepsilon \mu}$ the wavenumber. Here, $E_{P}:\left\{\mathbf{r}_{1}, \mathbf{r}_{2}, \mathbf{r}_{3}\right\}$ and $E_{Q}:\left\{\mathbf{r}_{1}, \mathbf{r}_{4}, \mathbf{r}_{5}\right\}$ correspond to the vertex adjacent triangular elements of the mesh (see Fig. 1), as dictated by the "element-by-element" approach of object-oriented programming. The above-mentioned integrals can be categorized into weakly singular (1) and (2) and strongly singular (3), respectively. Moreover, one can easily replace RWG functions with the novel Buffa-Cristiansen functions [10] without affecting the overall mathematical complexity since the latter are linear combinations of the former on the barycentric mesh. Finally, smart usage of both functions can lead to well-tested SIE operators, improving the overall accuracy of SIE solvers, provided of course that the associated impedance matrix elements are accurately computed.

\section{Direct Evaluation}

In the case of vertex adjacent triangles, the 2-D integrals for the observation and source elements are separately finite, the singularity being limited to a single point in the 4-D integration. Although there is a variety of methods that can be used to evaluate these terms, their overall accuracy cannot meet the requirements for the state-of-the-art SIE formulations. Here, we resort to the direct evaluation method and present an extension specially tailored to weakly singular and strongly singular integrals (1)-(3), arising when observation and source triangles share only one point.

As explained in detail in [4]-[8], the first step is to introduce an equilateral parameter space $\{\eta, \xi\}$, where $-1 \leq \eta \leq 1,0 \leq$ $\xi \leq \sqrt{3}(1-|\eta|)$, for each one of the triangles and orient the elements so that the singular point is $\eta=-1$ and $\eta^{\prime}=-1$. The singular integrals of interest in the new parametric space read as

$$
I=J \int_{-1}^{1} d \eta \int_{0}^{\xi(\eta)} d \xi \int_{-1}^{1} d \eta^{\prime} \int_{0}^{\xi\left(\eta^{\prime}\right)} d \xi^{\prime}
$$

where $\xi(\eta)=\sqrt{3}(1-|\eta|)$ and $J=A_{p} A_{q} / 3$ is the Jacobian of the transformation. Note also that the various integrands are omitted throughout this manuscript due to paucity of space, but essential information regarding their different nature is given when needed.

Next, we introduce the following separate polar coordinate systems for each element [see Fig. 2(a)]:

$$
\begin{aligned}
\eta^{\prime}=\rho_{q} \cos \left(\theta_{q}\right)-1 & \xi^{\prime}=\rho_{q} \sin \left(\theta_{q}\right) \\
\eta=\rho_{p} \cos \left(\theta_{p}\right)-1 & \xi=\rho_{p} \sin \left(\theta_{p}\right) .
\end{aligned}
$$

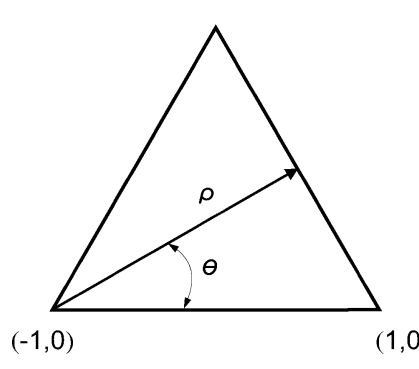

(a)

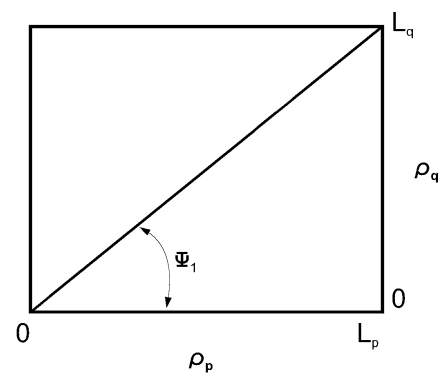

(b)
Fig. 2. Polar coordinate transformations employed in the vertex adjacent integration: (a) First transformation: $\{\eta, \xi\} \rightarrow\left\{\rho_{p}, \theta_{p}\right\}$ and $\left\{\eta^{\prime}, \xi^{\prime}\right\} \rightarrow\left\{\rho_{q}, \theta_{q}\right\}$, (b) Second transformation: $\left\{\rho_{p}, \rho_{q}\right\} \rightarrow\{\Lambda, \Psi\}$.

This results in an integral of the form (omitting the Jacobian)

$$
I=\int_{0}^{\pi / 3} d \theta_{p} \int_{0}^{L\left(\theta_{p}\right)} d \rho_{p} \int_{0}^{\pi / 3} d \theta_{q} \int_{0}^{L\left(\theta_{q}\right)} d \rho_{q}
$$

which, after a reordering of the integration, boils down to the following:

$$
I=\int_{0}^{\pi / 3} d \theta_{p} \int_{0}^{\pi / 3} d \theta_{q} \int_{0}^{L\left(\theta_{p}\right)} d \rho_{p} \int_{0}^{L\left(\theta_{q}\right)} d \rho_{q}
$$

where

$$
L\left(\theta_{p, q}\right)=\frac{2 \sqrt{3}}{\sin \left(\theta_{p, q}\right)+\sqrt{3} \cos \left(\theta_{p, q}\right)} .
$$

It is worth mentioning at this point that the reordering is a keypoint of the direct evaluation method and is actually the feature that differentiates it among the various singularity cancellation schemes. Upon this change, the 4-D integral is no more a series of 2-D integrals for the observation and source domains and the integrand is further regularized, in addition to the singularity cancellation.

The singularity is now located at the common vertex $\rho_{p}=\rho_{q}=0$, justifying the use of one further polar coordinate transformation

$$
\rho_{p}=\Lambda \cos (\Psi) \quad \rho_{q}=\Lambda \sin (\Psi) .
$$

After the new transformation, the distance vector function takes the form

$$
\mathbf{R}=\mathbf{r}-\mathbf{r}^{\prime}=\Lambda \boldsymbol{\Gamma}\left(\theta_{p}, \theta_{q}, \Psi\right)
$$

where

$$
\begin{aligned}
\boldsymbol{\Gamma}\left(\theta_{p}, \theta_{q}, \Psi\right)= & {\left[\boldsymbol{\alpha}_{v_{1}} \cos \left(\theta_{p}\right)+\boldsymbol{\alpha}_{v_{2}} \sin \left(\theta_{p}\right)\right] \cos (\Psi) } \\
& -\left[\boldsymbol{\alpha}_{v_{3}} \cos \left(\theta_{q}\right)+\boldsymbol{\alpha}_{v_{4}} \sin \left(\theta_{q}\right)\right] \sin (\Psi)
\end{aligned}
$$

and

$$
\begin{aligned}
& \boldsymbol{\alpha}_{v_{1}}=\left(\mathbf{r}_{\mathbf{2}}-\mathbf{r}_{\mathbf{1}}\right) / 2 \\
& \boldsymbol{\alpha}_{v_{2}}=\left(2 \mathbf{r}_{\mathbf{3}}-\mathbf{r}_{\mathbf{1}}-\mathbf{r}_{\mathbf{2}}\right) / 2 \sqrt{(3)} \\
& \boldsymbol{\alpha}_{v_{3}}=\left(\mathbf{r}_{\mathbf{4}}-\mathbf{r}_{\mathbf{1}}\right) / 2 \\
& \boldsymbol{\alpha}_{v_{4}}=\left(2 \mathbf{r}_{\mathbf{5}}-\mathbf{r}_{\mathbf{1}}-\mathbf{r}_{\mathbf{4}}\right) / 2 \sqrt{(3)} .
\end{aligned}
$$


As indicated in Fig. 2(b), the $\left\{\rho_{p}, \rho_{q}\right\}$ domain is a rectangle and the $\Psi$ integration must be taken in two pieces, as follows:

$$
\begin{aligned}
I=\int_{0}^{\pi / 3} d \theta_{p} \int_{0}^{\pi / 3} d \theta_{q} \\
\cdot\left[\int_{0}^{\Psi_{1}} d \Psi \int_{0}^{L_{1}} d \Lambda+\int_{\Psi_{1}}^{\pi / 2} d \Psi \int_{0}^{L_{2}} d \Lambda\right]
\end{aligned}
$$

where

and

$$
L_{1}=\frac{L\left(\theta_{p}\right)}{\cos (\Psi)} \quad L_{2}=\frac{L\left(\theta_{q}\right)}{\sin (\psi)}
$$

$$
\Psi_{1}=\tan ^{-1}\left(\frac{L\left(\theta_{q}\right)}{L\left(\theta_{p}\right)}\right)
$$

The combined Jacobian after the whole set of parametric transformations is given by

$$
\mathcal{J}=\frac{A_{p} A_{q}}{3} \Lambda^{3} \sin (\Psi) \cos (\Psi)
$$

while the singular part of the integrals can be written as

$$
\begin{aligned}
G(R) & =\frac{e^{-j k \Lambda \Gamma}}{\Lambda \Gamma} \\
\nabla G(R) & =-\frac{e^{-j k \Lambda \Gamma}}{\Lambda^{2} \Gamma^{3}}(1+j k \Lambda \Gamma) \Gamma .
\end{aligned}
$$

Obviously, both singularities, i.e., weakly singular $1 / \Lambda$ and strongly singular $1 / \Lambda^{2}$, are canceled by the overall Jacobian $\mathcal{J}$. In fact, it is easy to prove that for both RWG and $\hat{\mathbf{n}} \times$ RWG basis/testing functions under study, the overall integrands consist solely of terms proportional to $\Lambda^{s} e^{-j k \Lambda \Gamma\left(\theta_{p}, \theta_{q}, \Psi\right)}$, for $s=1,2,3,4$, which admit analytical solution with respect to $\Lambda$. Hence, the various integrands for the singular integrals (1)-(3) are given by

$$
\mathcal{F}^{1,2}\left(\theta_{p}, \theta_{q}, \Psi\right)=\sum_{s \in[1,4]} c_{s}\left(\theta_{p}, \theta_{q}, \Psi\right) K_{s}^{1,2}\left(\theta_{p}, \theta_{q}, \Psi\right)
$$

where

$$
K_{s}^{1,2}\left(\theta_{p}, \theta_{q}, \Psi\right)=\int_{0}^{L_{1,2}} \Lambda^{s} e^{-j k \Lambda \Gamma\left(\theta_{p}, \theta_{q}, \Psi\right)} d \Lambda
$$

and the coefficients $c_{s}\left(\theta_{p}, \theta_{q}, \Psi\right)$ can be systematically evaluated with the help of a symbolic mathematical software like Maple and exported to both MATLAB and $C$ platforms. We note also that a possible incorporation of high-order basis/testing functions over planar triangular elements only calls for a minor modification of the Maple codes, while the case of curvilinear triangles needs a more elaborate treatment as evinced by our ongoing studies. Finally, the singular integrals under investigation are given by

$$
I=\int_{0}^{\pi / 3} d \theta_{p} \int_{0}^{\pi / 3} d \theta_{q}\left[\int_{0}^{\Psi_{1}} d \Psi+\int_{\Psi_{1}}^{\pi / 2} d \Psi\right]
$$

where the integrands (19) are sufficiently smooth functions with respect to all three variables of the remaining 3-D integrals, allowing straightforward integration via simple Gauss-Legendre quadrature rules readily available in the literature.

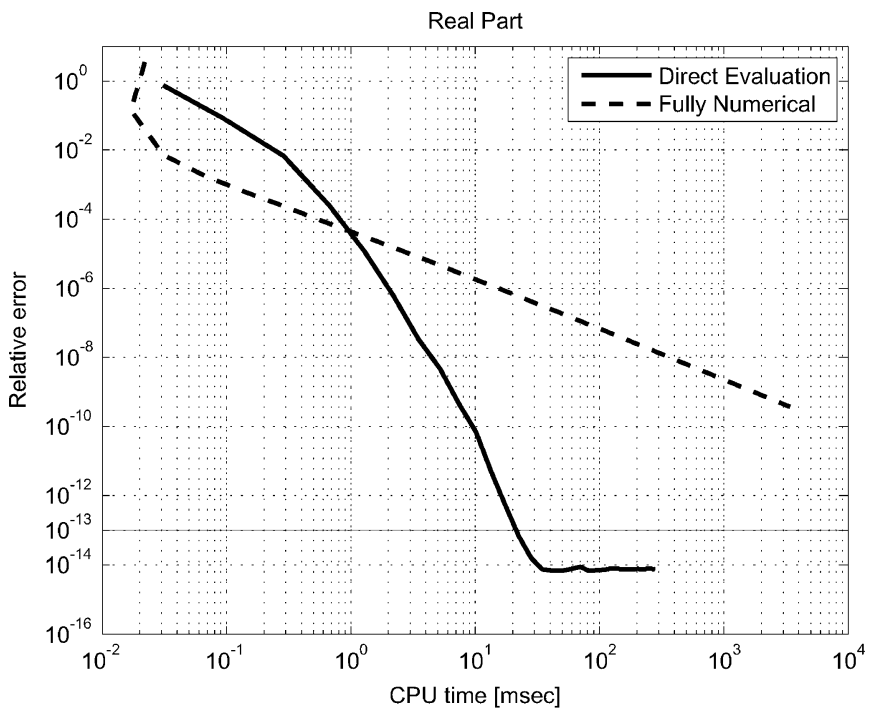

Fig. 3. Comparison of the maximum relative error as a function of the CPU time for the weakly singular integrals (1) and (2) over triangles T1 and T2, incorporating RWG basis and testing functions.

\section{Numerical Results}

This section presents examples that demonstrate the superior behavior of the proposed direct evaluation method and highlight the importance of incorporating such a sophisticated approach even for the case of SIE impedance matrix elements involving point singularities. More specifically, we designed an elaborate numerical experiment involving all singular integrals under study. For the case of weakly singular integrals (1) and (2), we consider RWG basis/testing functions in (2) and two vertex adjacent triangles (T1: $\left\{\mathbf{r}_{1}, \mathbf{r}_{2}, \mathbf{r}_{3}\right\}$ and $\left.\mathrm{T} 2:\left\{\mathbf{r}_{1}, \mathbf{r}_{4}, \mathbf{r}_{5}\right\}\right)$ with the following vertices:

$$
\begin{aligned}
& \mathbf{r}_{1}=\left[d_{1}, d_{1}, d_{1}\right] \quad \mathbf{r}_{2}=\left[d_{2}, d_{1}, d_{1}\right] \quad \mathbf{r}_{3}=\left[d_{1}, d_{2}, d_{1}\right] \\
& \mathbf{r}_{4}=\left[0, d_{1}, d_{1}\right] \quad \mathbf{r}_{5}=\left[0, d_{2}, d_{1}\right]
\end{aligned}
$$

where $d_{1}=0.1 \lambda, d_{2}=0.2 \lambda$, and $\lambda=1[\mathrm{~m}]$ corresponds to the wavelength. In the case of strongly singular integrals (3), we consider both RWG and $\hat{\mathbf{n}} \times$ RWG testing functions together with RWG basis functions over two vertex adjacent triangles lying on orthogonal planes, i.e., T1 : $\left\{\mathbf{r}_{1}, \mathbf{r}_{2}, \mathbf{r}_{3}\right\}$ and T3 : $\left\{\mathbf{r}_{1}, \mathbf{r}_{6}, \mathbf{r}_{7}\right\}$, with

$$
\mathbf{r}_{6}=\left[0, d_{1}, d_{2}\right] \quad \mathbf{r}_{7}=\left[0, d_{2}, d_{2}\right] .
$$

We timed the proposed method against a fully numerical scheme based on generalized Cartesian product rules. All coupling integrals [1 for (1), 9 for (2) and (3), except the case of strongly singular integrals with RWG basis/testing functions where 1 out of 9 interactions vanishes identically] have been computed 1000 times each, and the overall run times have been averaged. The comparison was carried out using codes implemented in $\mathrm{C+}+$ with double precision arithmetic and run on a computer with Intel Core 2 Duo, $3 \mathrm{GHz}$ (no parallelization), Windows 7 Pro and Microsoft Visual Studio 2010.

Figs. 3 and 4 depict the maximum relative error of the real (singular portion) parts among the associated coupling integrals as a function of the overall CPU time [total time for the computation of all 10, 8, and 9 integrals as shown in Figs. 3, 4(a), and (b), respectively] for the benchmark cases described above. By 


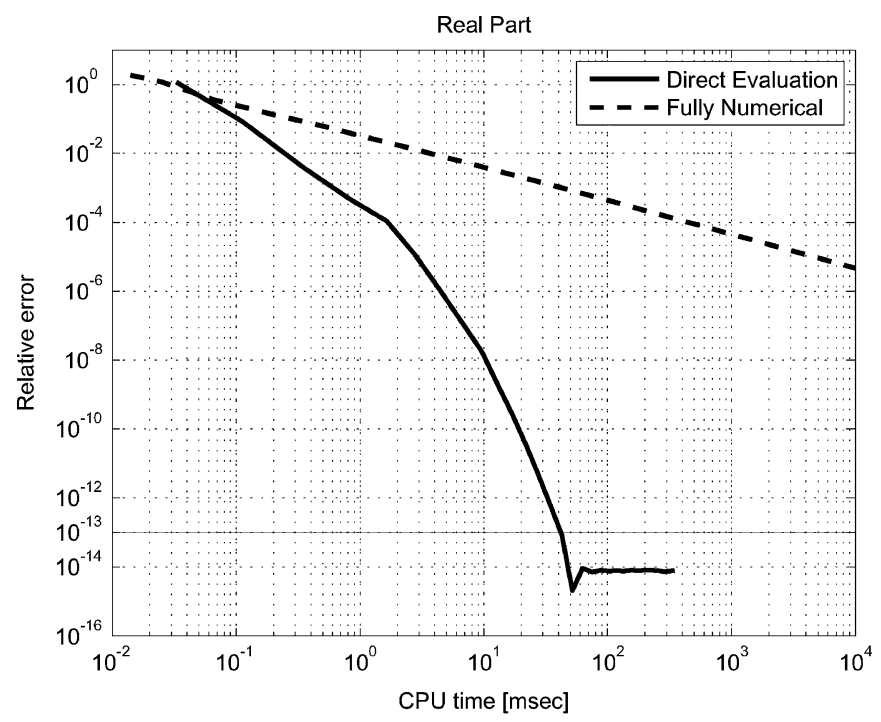

(a)

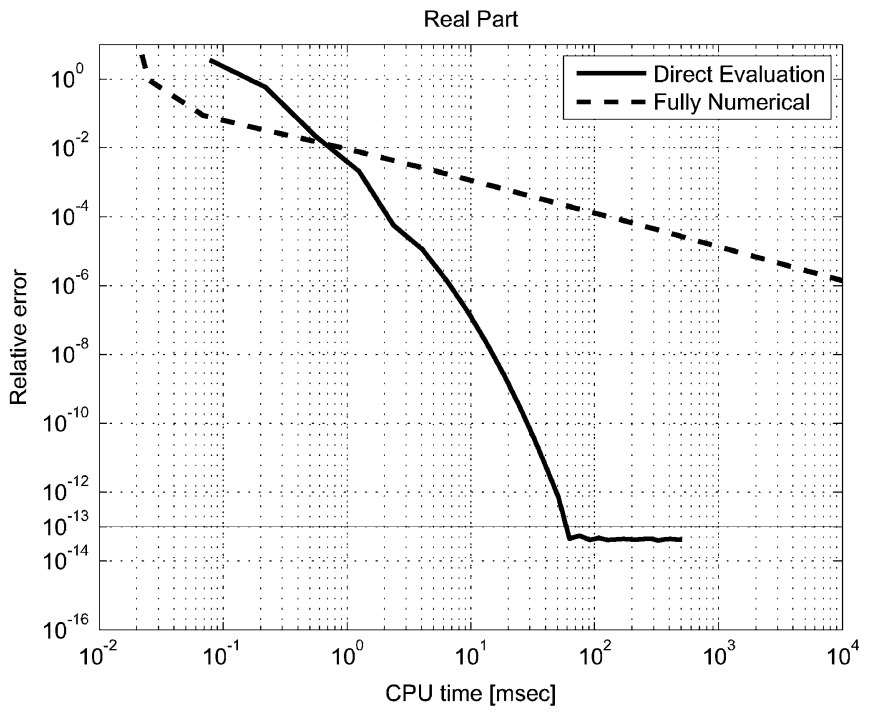

(b)

Fig. 4. Comparison of the maximum relative error as a function of the CPU time for the strongly singular integrals (3) over triangles T1 and T3. (a) RWG basis and testing functions. (b) RWG basis and $\hat{\mathbf{n}} \times$ RWG testing functions.

following [2], we assume a result to be numerically exact if its relative error is smaller than $10^{-13}$, thus, accounting for the incidental presence of error propagation effects in the numerical integrations. Moreover, the reference solutions have been obtained by means of the direct evaluation method using high-precision arithmetic in all computations ( $g++$ v.4.4.0 using long double representation of floating point numbers, which in turn provides machine epsilon $\approx 10^{-19}$ ) together with a high number of integration points, and they can be safely considered to be exact for double-precision arithmetic. According to these results, the proposed method provides numerically exact values for both weakly singular and strongly singular integrals and converges much faster than the fully numerical scheme, which is used traditionally (together with cancellation schemes) for the vertex adjacent integrals. The slightly slower convergence of the direct evaluation method for the strongly singular integrals (especially with $\hat{\mathbf{n}} \times$ RWG testing functions) is due to the higher complexity of the associated integrands in the final 3-D integration.

Additional numerical experiments undertaken by the authors have shown that the use of symmetrical Gaussian quadratures proposed in [11] cannot significantly improve the performance of the fully numerical scheme. Moreover, it is hard to get symmetrical rules of very high order as in the case of standard Gauss-Legendre rules. For instance, the limiting results of the fully numerical scheme have been obtained by utilizing $64^{4}$ integration points. Finally, the use of traditional singularity cancellation schemes for the case of weakly singular integrals did not seem to result in significant improvements. As for the strongly singular integrals, to the best of our knowledge, there is no robust cancellation scheme available in the literature.

\section{CONCLUSION}

One of the main error sources of the surface integral equation formulations, attributed to the evaluation of weakly singular and strongly singular 4-D integrals over vertex adjacent triangles, is successfully tackled by means of the direct evaluation method. The proposed scheme results in the straightforward integration of smooth 3-D integrals, and its overall performance in terms of accuracy and efficiency clearly outperforms the traditional fully numerical methods. This letter concludes our current investigations on the direct evaluation method for the evaluation of singular integrals arising in traditional surface integral equation formulations.

\section{REFERENCES}

[1] M. A. Khayat and D. R. Wilton, "Numerical evaluation of singular and near-singular potential integrals," IEEE Trans. Antennas Propag., vol. 53, no. 10, pp. 3180-3190, Oct. 2005.

[2] R. D. Graglia and G. Lombardi, "Machine precision evaluation of singular and nearly singular potential integrals by use of Gauss quadrature formulas for rational functions," IEEE Trans. Antennas Propag., vol. 56, no. 4, pp. 981-998, Apr. 2008.

[3] J. S. Asvestas, S. P. Yankovich, and O. E. Allen, "Calculation of the impedance matrix inner integral to prescribed accuracy," IEEE Trans. Antennas Propag., vol. 58, no. 2, pp. 479-487, Feb. 2010.

[4] L. J. Gray, J. M. Glaeser, and T. Kaplan, "Direct evaluation of hypersingular Galerkin surface integrals," SIAM J. Sci. Comput., vol. 25, no. 5, pp. 1534-1556, 2004.

[5] L. J. Gray, A. Salvadori, A. V. Phan, and A. Mantic, "Direct evaluation of hypersingular Galerkin surface integrals. II," Electron. J. Boundary Elements, vol. 4, no. 3, pp. 105-130, 2006.

[6] A. G. Polimeridis and T. V. Yioultsis, "On the direct evaluation of weakly singular integrals in Galerkin mixed potential integral equation formulations," IEEE Trans. Antennas Propag., vol. 56, no. 9, pp. 3011-3019, Sep. 2008.

[7] A. G. Polimeridis and J. R. Mosig, "Complete semi-analytical treatment of weakly singular integrals on planar triangles via the direct evaluation method,' Int. J. Numer. Meth. Eng., vol. 83, pp. 1625-1650, 2010.

[8] A. G. Polimeridis, J. M. Tamayo, J. M. Rius, and J. R. Mosig, "Fast and accurate computation of hypersingular integrals in Galerkin surface integral equation formulations via the direct evaluation method," IEEE Trans. Antennas Propag., vol. 59, no. 6, pp. 2329-2340, Jun. 2011.

[9] S. M. Rao, D. R. Wilton, and A. W. Glisson, "Electromagnetic scattering by surfaces of arbitrary shape," IEEE Trans. Antennas Propag., vol. 30, no. 5, pp. 409-418, May 1982.

[10] A. Buffa and S. H. Christiansen, "A dual finite element complex on the barycentric refinement," Math. Comput., vol. 76, no. 260, pp. 1743-1769, 2007.

[11] D. A. Dunavant, "High degree efficient symmetrical Gaussian quadrature rules for the triangle," Int. J. Num. Meth. Eng., vol. 21, pp. 1129-1148, 1985. 